\title{
Análisis topológico de los cables en un modelo de torre atirantada
}

\author{
Topological analysis of cables in a model of guyed tower
}

Fecha de entrega: 17 de junio 2019

Fecha de aceptación: 23 de agosto 2019

\section{Vivian B. Elena, Patricia Martín y Katia Luis}

Facultad de Ingeniería, Universidad Tecnológica de La Habana José Antonio Echevarría CUJAE, Calle 114, No. 11901, Ciclovía y Rotonda, Marianao, La Habana, Cuba, vivian@civil.cujae.edu.cu, patriciamr@civil.cujae.edu.cu, katial@civil.cujae.edu.cu

Las torres atirantadas son estructuras ampliamente empleadas en el área de las telecomunicaciones para soportar antenas a grandes alturas. Estas estructuras son sensibles a las cargas de viento y presentan un índice de fallo elevado frente al paso de fuertes vientos. En el comportamiento estructural de estas torres intervienen diversos factores como la topología de los cables (cantidad de cables en la torre, cantidad de cables por anclaje, ángulo de los cables con relación al fuste), la tensión de los cables en el momento de actuar la carga de viento y también la presencia de sistema reductores de la torsión, llamados antitorsores. En este trabajo se investiga mediante modelos de elementos finitos en SAP2000, el efecto que ejercen la topología de los cables y la presencia de los sistemas de reducción de torsión (antitorsores) a diferentes alturas en el comportamiento estructural de un modelo típico de torre atirantada, teniendo en cuenta las características dinámicas de la carga de viento, así como la no-linealidad geométrica de la estructura.

Palabras clave: torre atirantada, antitorsor, cables, nolinealidad, viento
Guyed towers are structures commonly used in the area of telecommunications for support antennas at high altitudes. The failure rate of this structural type with respect to others of similar complexity is high. Many factors are involved in the structural behaviour under the action of extreme winds. Cable topology (number of cables in the shaft, angle of cables respect to the shaft, number or anchors), tension of cables and torsion reduction systems are very significant in the response of these structures to wind forces. This study aims to investigate the effect of the topology of the cables and the torsion reduction system on the structural behaviour of a typical tower model using the finite element method with the SAP2000 software, taking into account the dynamic characteristics of the wind load and the non-linearity of the structure.

Keywords: guyed tower, star mounts, cables, nonlinearity, wind

\section{Introducción}

Las torres de celosía son estructuras comúnmente empleadas en la industria de las telecomunicaciones, las cuales tienen como objetivo soportar antenas a grandes alturas. Estas estructuras surgen en la segunda década del siglo XX con el nacimiento de las transmisiones de radio y con la invención de la televisión, su uso es extendido como soporte de antenas a partir de la década del 50 del mismo siglo. El número de colapsos de las torres de telecomunicaciones con respecto a otras estructuras de similar complejidad es elevado (Magued et al., 1989; Roitshtein, 1999; Smith, 2007; Travanca et al., 2013). Entre las principales causas de fallo de estas estructuras está la acción del viento y la rotura de los cables, lo cual ha motivado numerosas investigaciones en torno a su comportamiento estructural frente a la acción del viento
(Davenport, 1995; Peil et al., 1996; Harikrishna et al., 2003; LeBlanc Bakmar, 2004; Zhu, 2007; Páez y Sensale, 2017; Fernández et al., 2018; Alshurafa et al., 2019; Cobles et al., 2009). La Figura 1 muestra ejemplos de caídas de torres de telecomunicaciones.

Los cables son elementos fundamentales de una torre atirantada que provocan un comportamiento no lineal geométrico complejo, por lo que una parte importante de los estudios realizados a nivel internacional se han enfocado en el comportamiento de los cables y su interacción con la estructura, especialmente frente a la acción de las cargas dinámicas como el viento (Peil, 1993; Kewaisy, 2001; Lazzari et al., 2001; Andreu et al., 2006; Sparling y Wegner, 2007; Clobes y Peil, 2011; Elena Parnás et al., 2013; Shi y Salim, 2015; Pezo et al., 2016; Ballaben et al., 2017a,b; Hamada et al., 2017). 

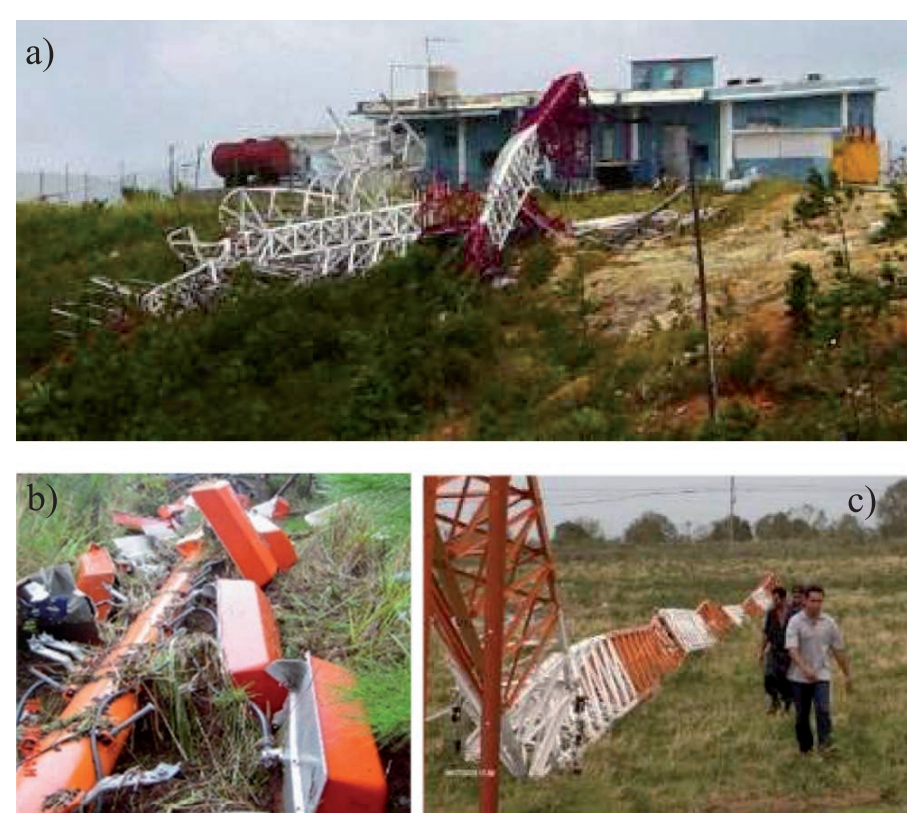

Figura 1: Fallo de torres de telecomunicaciones, Pinar del Río, Cuba: a) colapso total, b) presencia de antenas y c) fractura del fuste

La configuración o topología de cables de cada torre es variable, dado por una diferente cantidad de cables, diámetro, posición y ángulo de inclinación de los cables. Otro aspecto que ha sido estudiado por su repercusión en el diseño de las torres es la presencia de las antenas, pues generalmente estas se comportan como pantallas frente al viento que generan fuerzas y torsiones adicionales sobre la estructura. El desplazamiento y giro excesivo de la estructura puede provocar la pérdida de señal de la transmisión. Para contrarrestar este efecto, es común utilizar sistemas de reducción de torsión (denominados antitorsores o star-mounts en inglés) los cuales, colocados a diferentes alturas de la estructura, limitan el giro de la sección tal como se muestra en la Figura 2 (Kahla, 1995; Elena Parnás et al., 2013).

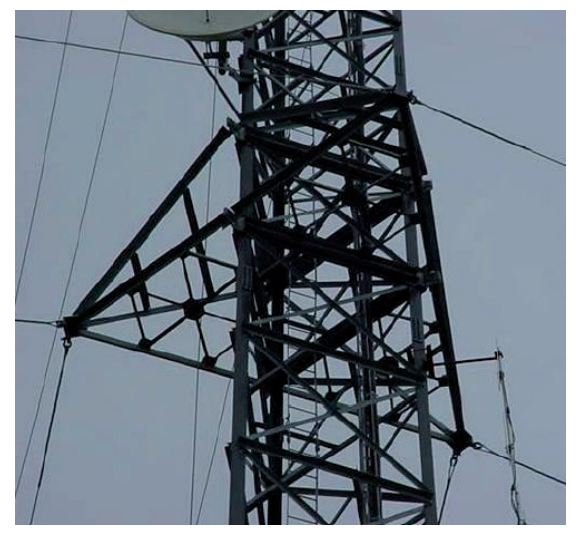

Figura 2: Imagen de antitorsor
Este trabajo se propone investigar el efecto que ejercen la topología de los cables variando tres factores: el número de cables, el diámetro de los cables y la cantidad de cables por anclaje. Además, se estudia la cantidad y posición de antitorsores en una torre atirantada sometida a carga de viento extrema aplicando el método de análisis no lineal quasi-estático de tramo de cargas.

\section{Materiales y métodos}

El modelo de torre escogido está compuesto por un fuste formado por elementos columnas, diagonales y tranques y por un sistema de cables en tres direcciones radiales, los cuales proporcionan rigidez lateral a la torre. Los cables están anclados a tierra y se sujetan a la estructura a diferentes niveles (Figura 3). La sección transversal del fuste es triangular equilátera de ancho $1.2 \mathrm{~m}$. Las columnas de cada vértice están formadas por perfiles angulares de alas iguales de longitud $100 \mathrm{~mm}$ y $10 \mathrm{~mm}$ de espesor. Los elementos de arriostre horizontal, denominados en este trabajo tranques, se conforman de perfiles angulares de alas iguales de longitud $63 \mathrm{~mm}$ y $6 \mathrm{~mm}$ de espesor, estos elementos se encuentran espaciados cada un metro a lo largo de todo el fuste. Las diagonales cruzadas son de $1.56 \mathrm{~m}$ de largo constituidas por perfiles angulares de alas iguales de longitud $50 \mathrm{~mm}$ y de espesor $5 \mathrm{~mm}$, estos elementos están unidos entre sí por un perno en su centro. La altura total de la torre es de $120 \mathrm{~m}$. El fuste en la parte superior presenta un mástil de $6 \mathrm{~m}$ de altura de sección tubular de $20 \mathrm{~cm}$ de diámetro con un espesor de $0.7 \mathrm{~cm}$, en el cual están colocados paneles de antenas.

La torre presenta 7 niveles de cables. Los valores de su carga de rotura fueron tomados según el catálogo de los fabricantes. La configuración en planta de la torre con la ubicación de los anclajes según las direcciones radiales se muestra en la Figura 4.

Para la modelación computacional fue empleado el programa de elementos finitos SAP2000 (2016). La torre se modeló como estructura reticulada en tres dimensiones con columnas continuas y diagonales y tranques articulados. Los cables se modelaron con formulación catenaria, y se les impuso una tensión inicial del 10\% de la carga de rotura dada por el fabricante. Para tranques, diagonales y columnas, el acero de los perfiles se consideró con peso específico de $77 \mathrm{kN} / \mathrm{m}^{3}$, módulo de elasticidad igual a 
$200 \mathrm{GPa}$, coeficiente de Poisson de 0.3 y módulo cortante de $77 \mathrm{GPa}$. La tensión de fluencia considerada fue de 248 MPa y la tensión de rotura es $400 \mathrm{MPa}$. Para los cables se consideró una tensión mínima de fluencia de $1600 \mathrm{MPa}$ y un módulo de elasticidad de $2 \times 10^{5} \mathrm{MPa}$. Tanto para los elementos de la torre como para los cables se plantea la hipótesis del material trabajando en un rango elástico y lineal.

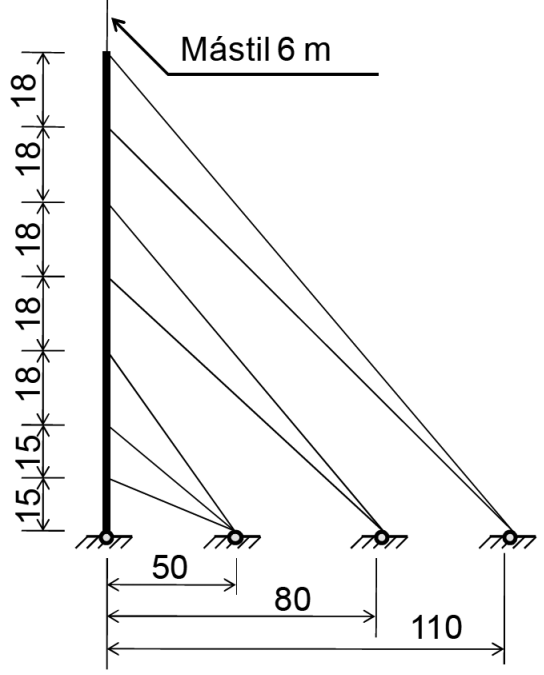

Figura 3: Elevación de la torre, unidades expresadas en metros

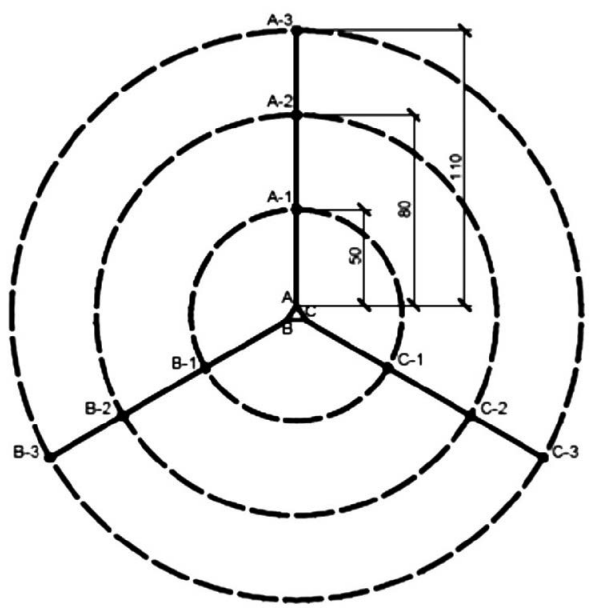

Figura 4: Configuración en planta de la torre, unidades expresadas en metros

Las acciones sobre la estructura se consideraron de acuerdo a la norma NC450 (2006) actuando como cargas el peso propio, la tensión de los cables y las cargas de viento, las cuales se combinaron como $1.2 D+1.4 W$ y $0.9 D+1.4 W$, siendo $D$ carga permanente y $W$ la carga de viento (presión básica $1.3 \mathrm{kN} / \mathrm{m}^{2}$ ). Se consideraron dos estados de análisis, uno inicial en donde a partir de la tensión inicial impuesta y el peso propio de todos los elementos, el programa genera la geometría deformada y obtiene la matriz de rigidez inicial y un segundo estado, que parte de esa geometría deformada e incorpora las cargas de viento.

Para la determinación de la carga de viento se utilizó el Método del Patch Load (Martín Rodríguez y Elena Parnás, 2011; Elena Parnás et al., 2013), el cual utiliza una serie de tramos de carga estáticos que son aplicados al fuste y usados para estimar la componente fluctuante. Los resultados de estas cargas por tramos, aplicadas individualmente sobre el fuste, se combinan y se suman a la componente media para obtener la respuesta dinámica de la estructura. Este método es específico para torres atirantadas y es propuesto por varias normas de torres de telecomunicaciones (CSA S37-01, 2001; TIA/EIA-222-G, 2005; EC 3: Part 3-1, 2007). Las direcciones del viento estudiadas fueron $0^{\circ}, 60^{\circ}$ y $90^{\circ}$ como recomiendan las normas para estas estructuras (Figura 5).

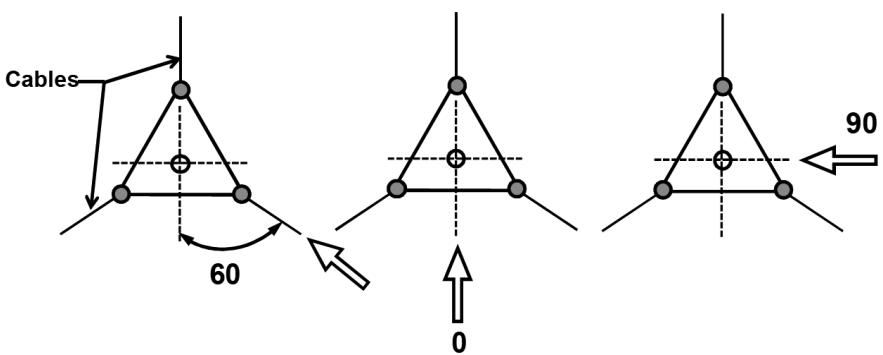

Figura 5: Direcciones de viento analizadas para el cálculo de la estructura

La carga de viento sobre los cables se consideró uniformemente distribuida, el valor se calculó teniendo en cuenta la velocidad básica y los coeficientes correspondientes a la mitad de la altura entre el anclaje del cable y su nivel de sujeción en el fuste. El coeficiente de forma para todos los cables se tomó igual a 1.2 según lo establecido por la NC285 (2003). La fuerza se aplicó en el sentido de la dirección de viento, teniendo en cuenta el ángulo del vector viento con el cable, según el caso de análisis.

Se realizó el análisis no lineal geométrico de la estructura a partir de dos estados de análisis dentro de los cuales se involucran todas las cargas a considerar. El primero es denominado estado inicial, corresponde al estado de equilibrio alcanzado para las cargas permanentes y tensión inicial de los cables. Este estado representa la fase final 
de la construcción de la torre (carga de peso propio más tensión inicial de los cables, sin carga ecológica). El segundo es el estado de equilibrio alcanzado para las cargas de viento sobre la estructura más las cargas que provoca la presencia de las antenas a partir de las condiciones alcanzadas en el estado previo (estado inicial). Para este estado, denominado estado final (estado inicial + carga de viento + peso propio antenas + viento sobre las antenas), las condiciones de partida del análisis no lineal fueron las condiciones finales del estado inicial, es decir, con los desplazamientos, tensiones, deformaciones y cargas del estado previo de trabajo.

\section{Variación en la topología de los cables}

La topología de los cables está dada por cuatro variables: la cantidad de cables, la cantidad de anclajes, la cantidad de cables que llegan a los anclajes y el diámetro de los cables. El análisis de la posición de los cables y anclajes quedó regido por el ángulo de los cables con respecto a la torre, quedando definidas entonces, tres variables independientes: cantidad de cables, cantidad de anclajes y diámetro de los cables. En el estudio se empleó un experimento factorial completo $2^{3} \mathrm{y}$ el procesamiento de los resultados se realizó usando el programa Statgraphics (statgraphics.net). La conjugación de las variables arrojó ocho modelos a analizar cuyas combinaciones de variables quedan definidas en la Figura 6 y Tabla1.

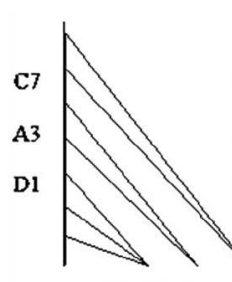

Modelo 1

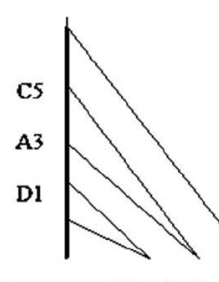

Modelo 5

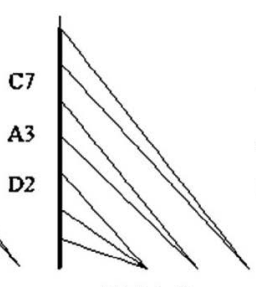

Modelo 2

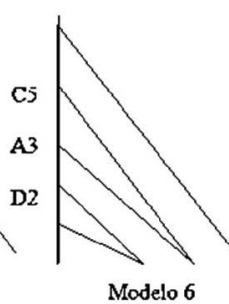

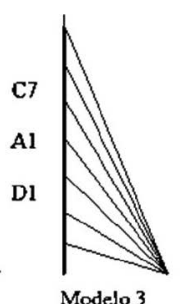

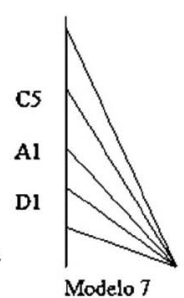

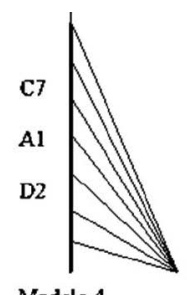

Modelo 4

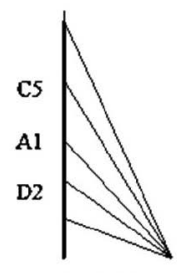

Madelo 8
Figura 6: Modelos analizados

Para la selección de los máximos y mínimos se tuvo en cuenta que, para el caso de la cantidad de cables se prevé que a menor cantidad de estos las fuerzas axiales de los elementos de la torre serán mayores; de igual forma, la disminución de la cantidad de anclajes aleja el ángulo de los cables de la condición ideal $\left(45^{\circ}\right)$ y por otra parte la reducción del diámetro de los cables reduce la rigidez estructural de la torre ante cargas horizontales. A partir de este análisis se obtuvo la Tabla 2.

Tabla 1: Matriz de simulación

\begin{tabular}{|c|c|c|c|c|c|c|}
\hline Modelo & $\begin{array}{c}\text { Cantidad } \\
\text { de cables } \\
\text { C }\end{array}$ & ID & $\begin{array}{c}\text { Cantidad } \\
\text { de anclajes } \\
\text { A }\end{array}$ & ID & $\begin{array}{c}\text { Diámetro de } \\
\text { cables D }\end{array}$ & ID \\
\hline 1 & 7 & C7 & 3 & A3 & $4 \Phi 19-3 \Phi 16$ & D1 \\
\hline 2 & 7 & C7 & 3 & A3 & $7 \Phi 16$ & D2 \\
\hline 3 & 7 & C7 & 1 & A1 & $4 \Phi 19-3 \Phi 16$ & D1 \\
\hline 4 & 7 & C7 & 1 & A1 & $7 \Phi 16$ & D2 \\
\hline 5 & 5 & C5 & 3 & A3 & $4 \Phi 19-3 \Phi 16$ & D1 \\
\hline 6 & 5 & C5 & 3 & A3 & $7 \Phi 16$ & D2 \\
\hline 7 & 5 & C5 & 1 & A1 & $4 \Phi 19-3 \Phi 16$ & D1 \\
\hline 8 & 5 & C5 & 1 & A1 & $7 \Phi 16$ & D2 \\
\hline
\end{tabular}

Tabla 2: Resumen Máximo y Mínimo de los niveles de las variables

\begin{tabular}{|l|c|c|}
\cline { 2 - 3 } \multicolumn{1}{c|}{} & \multicolumn{2}{c|}{ Niveles } \\
\cline { 2 - 3 } \multicolumn{1}{c|}{} & Máx. (1) & Mín. (-1) \\
\hline Cables & 5 & 7 \\
\hline Anclajes & 1 & 3 \\
\hline Diámetro (cables) & $7 \phi 16$ & $4 \phi 19-3 \phi 16$ \\
\hline
\end{tabular}

En la Tabla 2 se asocia el valor de 1 al nivel de las variables para las cuales se prevé un aumento de las solicitaciones debido a carga horizontal y el valor de -1 para los niveles en los cuales su incidencia se traducirá en decremento de las solicitaciones.

En la Figura 6 y Tabla 1, C representa la cantidad de cables, A la cantidad de anclajes y D las combinaciones de diámetro del cable (1 para el diseño típico 4\$19-3\$16 y 2 para todos cables de $16 \mathrm{~mm}$ ). Para el caso de los modelos con cinco niveles de cables, los dos primeros se consideraron con diámetro $16 \mathrm{~mm}$ y los restantes $19 \mathrm{~mm}$. En la Figura 4 el modelo 1 corresponde a los datos del modelo típico.

El estudio numérico tiene como objetivo fundamental identificar la influencia de las variables independientes y la interacción doble entre ellas, en los valores de las variables dependientes, en este caso fuerza axial en los elementos y desplazamiento de la torre. Para este objetivo se plantearon las siguientes hipótesis para cada una de las variables dependientes obtenidas: 
Ho: La variable independiente (A, B ó C) o la interacción de las variables (AB, BC ó $\mathrm{AC})$ no influyen el valor de la variable dependiente $x$ (fuerza axial y desplazamiento).

H1: La variable independiente (A, B ó C) o la interacción de las variables $(\mathrm{AB}, \mathrm{BC}$ ó $\mathrm{AC})$ influyen en el valor de la variable dependiente $x$ (fuerza axial y desplazamiento).

Donde A es la cantidad de cables, B es la cantidad de anclajes y $\mathrm{C}$ representa las combinaciones de los diámetros de cables.

Los resultados de este análisis están basados en la obtención del valor $p$ que indica la probabilidad de rechazar Ho cuando Ho es cierta para un nivel de significación de un $5 \%$. La regla de decisión seleccionada expone que si el valor $p$ es menor de 0.05 se rechaza Ho, si no se acepta. Por tanto, si los valores $p$ son menores que 0.05 se plantea que hay suficiente evidencia estadística como para rechazar Ho con un nivel de significación del $5 \%$, es decir, que hay suficiente evidencia estadística para rechazar que la variable independiente (A, B ó C) o la interacción de las variables ( $\mathrm{AB}, \mathrm{BC}$ ó $\mathrm{AC})$, según corresponda en cada caso, no influye en el valor de la variable dependiente $x$ con un nivel de significación del 5\%.

\section{Variación del número y posición de antitorsores}

Se analizaron cinco modelos numéricos de variantes de torre con posiciones diferentes de antitorsores, que fueron comparadas con el modelo sin antitorsores. A partir de las regularidades encontradas en los estudios de campo de la posición y número de los antitorsores. Para esta investigación se definieron 5 variantes: dos casos con un sólo antitorsor en el fuste en distinta posición (V1 uno en el primer tercio del fuste y V2 uno en el tope del fuste), dos casos con dos antitorsores en el fuste (V3 en el primer y segundo tercio del fuste, V4 en el segundo y tercer tercio del fuste) y un caso con tres antitorsores (V5 en el primer, segundo y tercer tercio del fuste) según se aprecia en la Figura 7.

El modelo de antitorsor usado para el análisis se muestra en la Figura 8 y está constituido por un conjunto de barras formando una celosía que se fija al fuste y de la cual salen seis cables en pares a los anclajes.

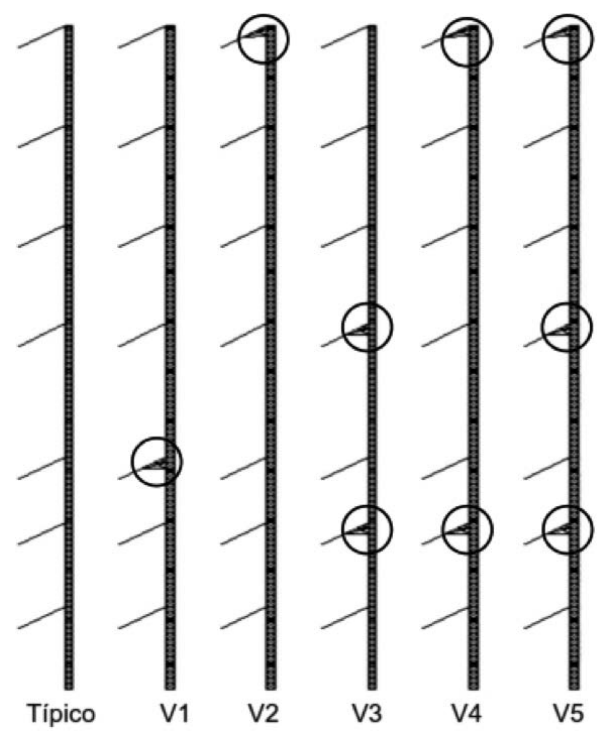

Figura 7: Modelos analizados con casos de estudio de antitorsor

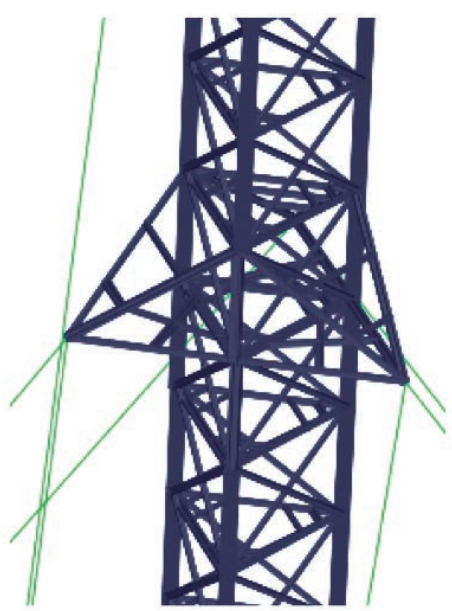

Figura 8: Esquema de antitorsor utilizado en la modelación

\section{Resultados de la variación en la topología de los cables}

Los resultados están basados en los valores de las fuerzas máximas obtenidas en los elementos de la torre: columnas, tranques, diagonales y cables y los desplazamientos máximos de la torre. En la Tabla 3 se muestran los valores $p$ obtenidos para las variables independientes (A, B ó C) y la interacción de las variables ( $\mathrm{AB}, \mathrm{BC}$ ó AC) para cada variable dependiente estudiada.

En el caso de las columnas y diagonales a tracción la única variable independiente que tiene influencia es la cantidad de cables. En los tranques a tracción los factores que muestran influencia significativa son cantidad de cables, cantidad de 
anclajes y la interacción entre ellos. Para los tranques a compresión se encuentran como variables independientes influyentes la cantidad de cables, diámetro de los cables y la interacción entre ellos. Finalmente, en el caso de los desplazamientos las tres variables independientes: cantidad de cables, cantidad de anclajes y diámetros de los cables, presentan influencia significativa. Todas estas afirmaciones se justifican con los $p$-valores obtenidos menores que 0.05 .

Tabla 3: Valores $p$ para las fuerzas axiales de los elementos y desplazamientos.

\begin{tabular}{|c|c|c|c|c|c|c|c|c|}
\hline Factores & $\begin{array}{c}\text { F. } \\
\text { Cables }\end{array}$ & $\begin{array}{c}\text { Col. } \\
\text { F. Trac. }\end{array}$ & $\begin{array}{c}\text { Col. } \\
\text { F.Comp. }\end{array}$ & $\begin{array}{c}\text { Tranq. } \\
\text { F.Trac. }\end{array}$ & $\begin{array}{c}\text { Tranq. } \\
\text { F.Comp. }\end{array}$ & $\begin{array}{c}\text { Diag. } \\
\text { F.Trac. }\end{array}$ & $\begin{array}{c}\text { Diag. } \\
\text { F.Comp. }\end{array}$ & Desplaz. \\
\hline $\begin{array}{c}\text { A: } \\
\text { Cantidad } \\
\text { de cables }\end{array}$ & 0.0588 & $\mathbf{0 . 0 2 3 8}$ & 0.1307 & $\mathbf{0 . 0 3 9 8}$ & $\mathbf{0 . 0 0 2 2}$ & $\mathbf{0 . 0 2 5 6}$ & 0.0805 & $\mathbf{0 . 0 4 8 9}$ \\
\hline $\begin{array}{c}\text { B: } \\
\text { Cantidad } \\
\text { de } \\
\text { anclajes }\end{array}$ & 0.0706 & 0.1469 & 0.0864 & $\mathbf{0 . 0 4 5 7}$ & 0.0886 & 0.8350 & 0.1145 & $\mathbf{0 . 0 1 3 6}$ \\
\hline $\begin{array}{c}\text { C: } \\
\text { Diámetro } \\
\text { de los } \\
\text { cables }\end{array}$ & 0.2591 & 0.2913 & 0.2754 & 0.0570 & $\mathbf{0 . 0 4 9 2}$ & 0.8759 & 0.9609 & $\mathbf{0 . 0 1 9 0}$ \\
\hline AB & 0.6419 & 0.1803 & 0.7523 & $\mathbf{0 . 0 2 7 0}$ & 0.0661 & 0.3484 & 0.2107 & 0.4546 \\
\hline AC & 0.8801 & 0.8084 & 0.5311 & 0.1016 & $\mathbf{0 . 0 3 6 8}$ & 0.2267 & 0.3900 & 0.3820 \\
\hline BC & 0.8930 & 0.5857 & 0.5433 & 0.0628 & 0.1804 & 0.3523 & 0.5587 & 0.1790 \\
\hline
\end{tabular}

a)

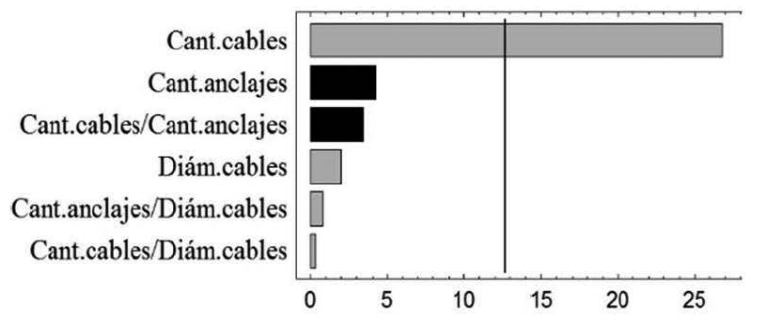

c)

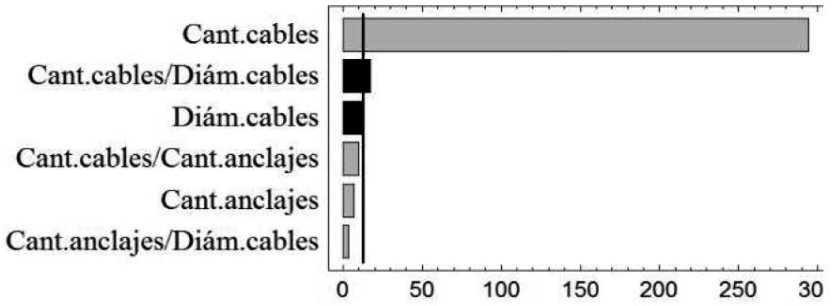

En los elementos donde se evidenció influencia de algunos factores se puede verificar gráficamente el orden de influencia de los mismos mediante los diagramas de Pareto mostrados en la Figura 9. En el diagrama de Pareto la línea vertical negra gruesa representa el nivel de significación del $5 \%$ por lo que todo factor que su efecto sobrepase la misma se puede afirmar que tiene influencia significativa en la variable dependiente (fuerzas axiales en los elementos o desplazamiento). La influencia de los factores se organiza de mayor a menor según muestran las barras y los signos indican la influencia creciente (barras grises) o decreciente (barras negras) en la variable dependiente.

Del análisis de los resultados de los modelos realizados se obtuvo que una disminución de la cantidad de anclajes de 3 a 1 provoca un incremento en las solicitaciones de los elementos del fuste de $22 \%$ para las columnas, $28 \%$ para los tranques y un $21 \%$ para las diagonales, así como un $34 \%$ de incremento sobre las fuerzas que actúan sobre los cables. Al disminuir la cantidad de cables de 7 a 5 se incrementaron las solicitaciones de los elementos del fuste en un $6 \%$ para las columnas, un 3\% para los tranques y un 13\% para las diagonales. Debido a la redistribución de los esfuerzos que aparece en la estructura aumentaron las fuerzas en los cables en un $37 \%$. En los modelos donde se

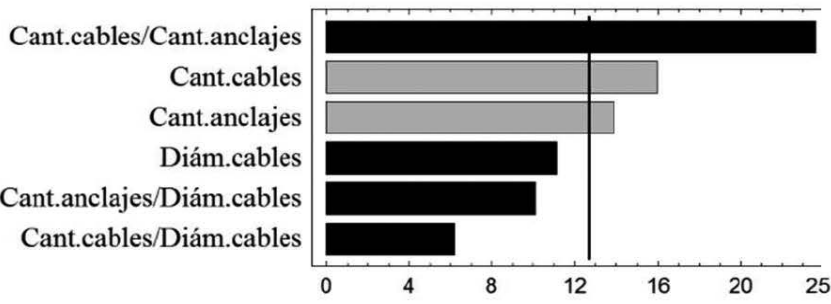

b)

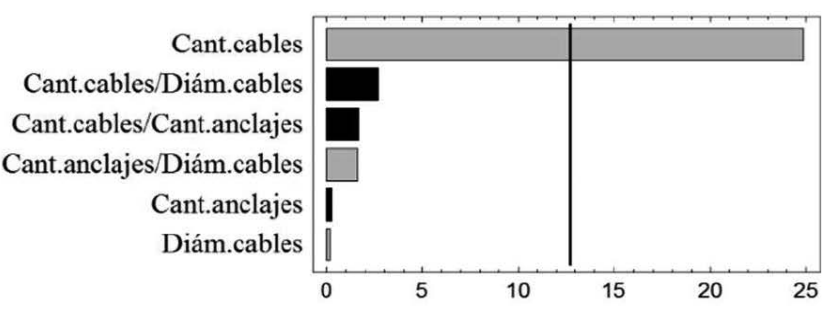

d)

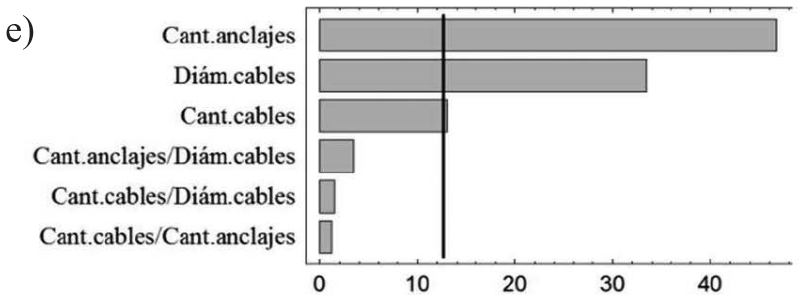

Figura 9: Diagramas de Pareto de las variables dependientes con influencia significativa de las variables independientes, a) columnas en tracción, b) tranques en tracción, c) tranques en compresión, d) diagonales en tracción y e) desplazamientos 
disminuyeron los diámetros de los cables, se produjeron variaciones en las solicitaciones del fuste. Las columnas sufrieron un incremento del $3 \%$, los tranques se mueven en el rango de un decremento del $4 \%$ hasta un incremento del $33 \%$ y las fuerzas en las diagonales se incrementaron en un $4 \%$. Para el caso de los cables, los incrementos fueron del $37 \%$

\section{Resultados de la variación del número y posición de los antitorsores}

Los resultados de la variación y posición de los antitorsores están basados en los valores de giro y desplazamiento de la torre en toda la altura. La Figura 10 muestra los valores de giros para cada una de las variantes estudiadas. Se compararon las variantes con un antitorsor (Figura 10a), con dos antitorsores (Figura 10b) y con tres antitorsores (Figura 10c) con respecto al modelo típico que no presenta antitorsor. Los resultados arrojaron que en los niveles donde aparece antitorsor se evidencia una reducción marcada del giro. En la Figura 10c se puede observar que la variante 5 con mayor número de antitorsores, resultó ser la que más redujo el giro máximo de la torre, que alcanzo el valor de $0.38^{\circ}$, el cual representa una reducción del giro en $81 \%$ con respecto al modelo típico sin antitorsor.

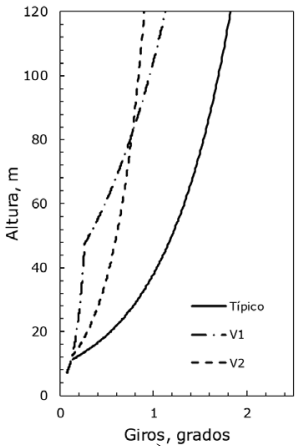

a)

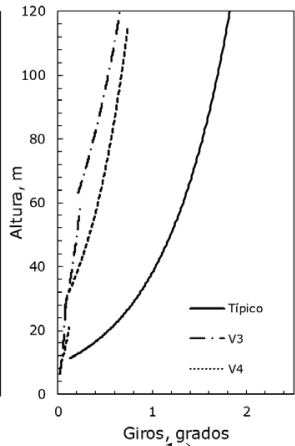

b)

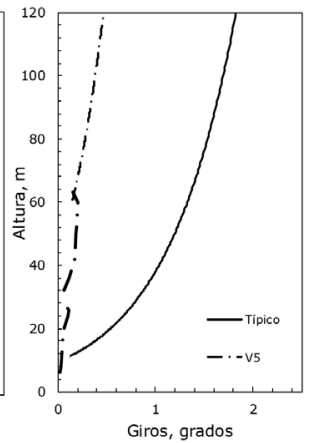

c)
Figura 10: Rotación en grados del fuste alrededor del eje vertical

En la Figura 11 se muestra el desplazamiento horizontal obtenido para las diferentes variantes analizadas. Los resultados arrojaron que las variantes con antitorsores colocados en el tope (V2, V4 y V5) de la torre fueron las que más redujeron el desplazamiento máximo en el tope.

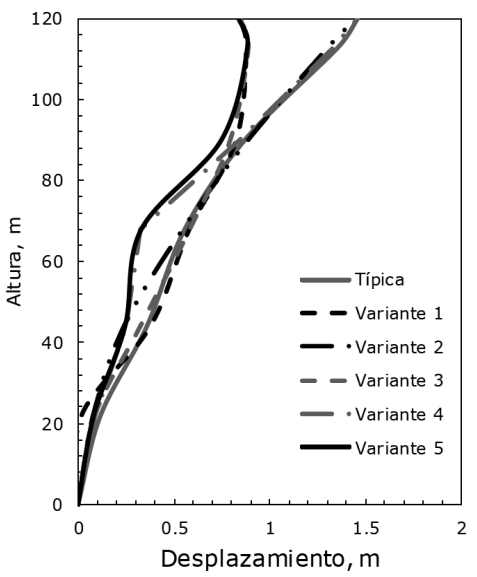

Figura 11: Desplazamientos horizontales para la dirección de viento perpendicular a una cara

\section{Conclusiones}

Los resultados del estudio numérico demostraron que sí existe influencia de la topología de cables en los valores de fuerza axial y desplazamiento de la torre estudiada. Este estudio demostró que las variables dependientes que presentan influencia significativa para un nivel de significación del $5 \%$, son las fuerzas axiales de tracción en los elementos columnas, diagonales y tranques, así como la fuerza axial en compresión de los tranques y los desplazamientos. La variable cantidad de cables tiene influencia en todas las variables dependientes y además es la más significativa en el $60 \%$ de los resultados.

La disminución del número de anclajes produjo incrementos en el rango entre $21-34 \%$ en las fuerzas axiales de los elementos de la torre. La disminución de la cantidad de cables y del diámetro de los cables generan incrementos en el rango $3-37 \%$ en los elementos de la torre, excepto en algunos de los tranques donde la disminución del diámetro de los cables provoca una disminución del $4 \%$ de la fuerza axial. Los antitorsores reducen el giro y el desplazamiento horizontal de la torre proporcional a la cantidad empleada y su efecto reductor se produce fundamentalmente en los tramos por debajo de la ubicación del mismo, mientras que los giros relativos entre secciones se mantienen iguales por encima de este. La posición de antitorsor que más redujo el giro de la torre fue en el tope de la misma. La colocación de antitorsores logró reducir el giro máximo en un $81 \%$ con respecto al modelo típico sin antitorsor. El desplazamiento horizontal máximo en el tope se logró reducir en un $40 \%$ para los casos de antitorsores en el tope. 


\section{Referencias}

Alshurafa, S., Alhayek, H. And Polyzois, D. (2019). Finite Element Method For The Static And Dynamic Analysis Of Frp Guyed Tower. Journal Of Computational Design And Engineering 6(3), 436-446

Andreu, A., Gil, L. and Roca, P. (2006). A new deformable catenary element for the analysis of cable net structures. Computers \& Structures 84(29-30), 1882-1890

Ballaben, J.S., Sampaio, R. and Rosales, M.B. (2017a). Uncertainty quantification in the dynamics of a guyed mast subjected to wind load. Engineering Structures 132, 456-470

Ballaben, J.S., Guzmán, A.M. and Rosales, M.B. (2017b). Nonlinear dynamics of guyed masts under wind load: sensitivity to structural parameters and load models. Journal of Wind Engineering and Industrial Aerodynamics 169, 128-138

CSA S37-01 (2001). Antennas, towers, and antenna supporting structures. Canadian Standards Association, Rexdale, Canada

Clobes, M. and Peil, U. (2011). Unsteady buffeting wind loads in the time domain and their effect on the life-cycle prediction of guyed masts. Structure and Infrastructure Engineering 7(1-2), 187-196

Clobes, M., Willecke, A. and Peil, U. (2009). A refined analysis of guyed masts in turbulent wind. Fifth European \& African Conferences on Wind Engineering EACWE 5, Florence, Italy

Davenport, A.G. (1995). The response of slender structures to wind. In Wind Climate in Cities, Cermak, J.E., Davenport, A.G., Plate, E.J. and Viegas, D.X. (eds.). Series E: Applied Sciences, vol. 277. Springer, Dordrecht, 209-239

EC 3: Part 3-1 (2007). Eurocode 3: Design of steel structures - Part 3-1: Towers, masts and chimeneys-towers and masts. Brussels, Belgium

Elena Parnás, V., Martín Rodríguez, P. and Castañeda Hevia, A.E. (2013). Structural behavior of guyed mast with asymmetrical anchors. Journal of the Brazilian Society of Mechanical Sciences and Engineering 35(2), 61-67

Fernández, I., Elena, V.B., Martín, P., Corona, Y. y Hernández, I.A. (2018). Análisis dinámico de una torre autosoportada sujeta a cargas de viento y sismo. Obras y Proyectos 23, 78-86
Hamada, A., King, J.P.C., El Damatty, A.A., Bitsuamlak, G. and Hamada, M. (2017). The response of a guyed transmission line system to boundary layer wind. Engineering Structures 139, $135-152$

Harikrishna, P., Annadurai, A. Gomathinayagam, S. and Lakshmanan, N. (2003). Full scale measurements of the structural response of a $50 \mathrm{~m}$ guyed mast under wind loading. Engineering Structures 25(7), 859-867

Kahla, N.B. (1995). Influence of star mounts on guyed towers. Computers \& Structures 54(5), 989-995

Kewaisy, T.H. (2001). Nonlinear dynamic interaction between cables and mast of guyed-tower systems subjected to windinduced forces. PhD thesis, Texas Tech University, USA.

Lazzari, M., Saetta, A.V. and Vitaliani, R.V. (2001). Non-linear dynamic analysis of cable-suspended structures subjectd to wind actions. Computers \& Structures 79(9), 953-969

LeBlanc Bakmar, C. (2004). Wind load on guyed mast. Master thesis, Technical University of Denmark

Magued, M.H., Bruneau, M. and Dryburgh, R.B. (1989). Evolution of design standards and recorded failures of guyed towers in Canada. Canadian Journal of Civil Engineering 16(5), $725-732$

Martín Rodriguez, P. and Elena Parnás, V. (2011). Simplified methods of dynamic analysis for guyed towers under the action of extreme winds. Revista Técnica de la Facultad de Ingeniería Universidad del Zulia 34(3), 185-193

NC450 (2006). Edificaciones-Factores de Carga o PonderaciónCombinaciones. Oficina Nacional de Normalización, La Habana, Cuba

NC285 (2003 ). Carga de viento. Método de cálculo. Oficina Nacional de Normalización, La Habana, Cuba

Páez, P.M. and Sensale, B. (2017). Analysis of guyed masts by the stability functions based on the Timoshenko beam-column. Engineering Structures 152, 597-606

Peil, U. (1993). Dynamic behaviour of guys. Meeting of IASS Working Group 4: Towers and Masts, Prague, Czech Republic

Peil, U., Nölle, H. and Wang, Z.H. (1996). Dynamic behaviour of guys under turbulent wind load. Journal of Wind Engineering and Industrial Aerodynamics 65(1-3), 43-54 
Pezo, M.L., Bakic, V.V. and Markovic, Z.J. (2016). Structural analysis of guyed mast exposed to wind action. Thermal Science 20(5), 1473-1483

Roitshtein, M.M. (1999). Analysis of masts and towers failures. IASS masts and towers working group meeting. Krakow, Poland SAP2000 (2016). Structural analysis program. Integrated software for structural analysis and design, v.16. Computers and Structures Inc., Berkeley, USA

Shi, H. and Salim, H. (2015). Geometric nonlinear static and dynamic analysis of guyed towers using fully nonlinear element formulations. Engineering Structures 99, 492-501

Smith, B.W. (2007). Communication structures. ThomasTelford, London, Great Britain
Sparling, B.F. and Wegner, L.D. (2007). Estimating peak wind load effects in guyed masts. Wind and Structures 10(4), 347-366

TIA/EIA-222-G (2005). Structural standards for steel antenna towers and antenna supporting structures. Telecommunications Industry Association, USA

Travanca, R., Varum, H. and Real, P.V. (2013). The past 20 years of telecommunication structures in Portugal. Engineering Structures 48, 472-485

Zhu, N. (2007). Wind tunnel test for guyed mast dynamic characteristics under wind loads. MSc thesis, University of Saskatchewan, Canada 